\title{
Entropy and the Law of Small Numbers
}

\author{
$\begin{array}{lll}\text { I. Kontoyiannis* } & \text { P. Harremoës } & \text { O. Johnson }\end{array}$
}

December 22, 2004

\begin{abstract}
Two new information-theoretic methods are introduced for establishing Poisson approximation inequalities. First, using only elementary information-theoretic techniques it is shown that, when $S_{n}=\sum_{i=1}^{n} X_{i}$ is the sum of the (possibly dependent) binary random variables $X_{1}, X_{2}, \ldots, X_{n}$, with $E\left(X_{i}\right)=p_{i}$ and $E\left(S_{n}\right)=\lambda$, then

$$
D\left(P_{S_{n}} \| \operatorname{Po}(\lambda)\right) \leq \sum_{i=1}^{n} p_{i}^{2}+\left[\sum_{i=1}^{n} H\left(X_{i}\right)-H\left(X_{1}, X_{2}, \ldots, X_{n}\right)\right]
$$

where $D\left(P_{S_{n}} \| \mathrm{Po}(\lambda)\right)$ is the relative entropy between the distribution of $S_{n}$ and the Poisson $(\lambda)$ distribution. The first term in this bound measures the individual smallness of the $X_{i}$ and the second term measures their dependence. A general method is outlined for obtaining corresponding bounds when approximating the distribution of a sum of general discrete random variables by an infinitely divisible distribution.

Second, in the particular case when the $X_{i}$ are independent, the following sharper bound is established,

$$
D\left(P_{S_{n}} \| \operatorname{Po}(\lambda)\right) \leq \frac{1}{\lambda} \sum_{i=1}^{n} \frac{p_{i}^{3}}{1-p_{i}},
$$

and it is also generalized to the case when the $X_{i}$ are general integer-valued random variables. Its proof is based on the derivation of a subadditivity property for a new discrete version of the Fisher information, and uses a recent logarithmic Sobolev inequality for the Poisson distribution.
\end{abstract}

Keywords: Poisson approximation, law of small numbers, convergence in relative entropy, Fisher information, total variation, logarithmic Sobolev inequality, subadditivity

\footnotetext{
*Division of Applied Mathematics and Dept of Computer Science, Brown Univ, 182 George St., Providence, RI 02912, USA. Email: yiannis@dam.brown.edu Web: www.dam.brown.edu/people/yiannis/. Supported in part by NSF grants \#0073378-CCR and DMS-9615444, and by USDA-IFAFS grant \#00-52100-9615.

${ }^{\dagger}$ Department of Mathematics, University of Copenhagen, Universitetsparken 5, DK-2100 København $\emptyset$, Denmark. Email: moes@math.ku.dk. Supported in part by a grant from the Cowi Foundation, and by a post-doctoral fellowship from the Villum Kann Rasmussen Foundation.

${ }^{\ddagger}$ Statistical Laboratory, Centre for Mathematical Sciences, Wilberforce Road, Cambridge CB3 0WB, U.K. Email: otj1000@cam.ac.uk.
} 


\section{Introduction}

Let $X_{1}, X_{2}, \ldots, X_{n}$ be binary random variables. A classical result in probability states that, if the $X_{i}$ are independent and identically distributed (i.i.d.) with common parameter $p_{i}=$ $E\left(X_{i}\right)=\lambda / n$, then, when $n$ is large, the distribution of their sum

$$
S_{n}=X_{1}+X_{2}+\cdots+X_{n}
$$

is close to $\operatorname{Po}(\lambda)$, the Poisson distribution with parameter $\lambda$. More generally, analogous results apply when the $X_{i}$ are possibly dependent and not necessarily identically distributed. The distribution of $S_{n}$ is close to $\operatorname{Po}(\lambda)$ as long as:

(a) The sum $\sum p_{i}$ of the parameters $p_{i}$ of the $X_{i}$ is close to $\lambda$.

(b) None of the $X_{i}$ dominate the sum, i.e., all the $p_{i}$ are small.

(c) The variables $X_{i}$ are not strongly dependent.

Such results are often referred to as "laws of small numbers" or "Poisson approximation results." See [1][17, Section 2.6][3] for details.

Our purpose here is to illustrate how techniques based on information-theoretic ideas can be used to establish general Poisson approximation inequalities. In Section 2 we prove:

Proposition 1. Poisson Approximation in Relative Entropy: If $S_{n}=\sum_{i=1}^{n} X_{i}$ is the sum of $n$ (possibly dependent) binary random variables $X_{1}, X_{2}, \ldots, X_{n}$ with parameters $p_{i}=E\left(X_{i}\right)$ and with $E\left(S_{n}\right)=\sum_{i=1}^{n} p_{i}=\lambda$, then the distribution $P_{S_{n}}$ of $S_{n}$ satisfies

$$
D\left(P_{S_{n}} \| \operatorname{Po}(\lambda)\right) \leq \sum_{i=1}^{n} p_{i}^{2}+\left[\sum_{i=1}^{n} H\left(X_{i}\right)-H\left(X_{1}, X_{2}, \ldots, X_{n}\right)\right] .
$$

For two probability distributions $P$ and $Q$ on a discrete set $S$, the relative entropy between $P$ and $Q$ is defined as $D(P \| Q)=\sum_{x \in S} P(x) \log \frac{P(x)}{Q(x)}$, and the entropy of a discrete random variable (or random vector) $X$ with distribution $P$ on $S$ is $H(X)=H(P)=$ $-\sum_{x \in S} P(x) \log P(x)$, where $\log$ denotes the natural logarithm.

Whenever $(a),(b)$ and $(c)$ hold we expect the two terms in the right-hand side of (1) to be small, and hence the distribution of $S_{n}$ to be close to $\operatorname{Po}(\lambda)$ in the relative entropy sense. Although $D(P \| Q)$ is not a proper metric, it is a natural measure of "dissimilarity" in the context of statistics [26][11, Ch. 12], and it can be used to define a topology on probability measures [20]. Also, bounds in relative entropy can be translated into bounds in total variation via Pinsker's inequality $[11]$

$$
\frac{1}{2}\|P-Q\|_{\mathrm{TV}}^{2} \leq D(P \| Q) .
$$

For example, if the $X_{i}$ are independent (1) reduces to

$$
D\left(P_{S_{n}} \| \operatorname{Po}(\lambda)\right) \leq \sum_{i=1}^{n} p_{i}^{2}
$$


Although this is reminiscent of the simple total-variation bound due to Le Cam [27],

$$
\left\|P_{S_{n}}-\operatorname{Po}(\lambda)\right\|_{\mathrm{TV}} \leq \sum_{i=1}^{n} p_{i}^{2}
$$

(which, incidentally, only holds when the $X_{i}$ are independent), applying Pinsker's inequality (2) to (3) leads to the suboptimal bound

$$
\left\|P_{S_{n}}-\operatorname{Po}(\lambda)\right\|_{\mathrm{TV}} \leq\left[2 \sum_{i=1}^{n} p_{i}^{2}\right]^{1 / 2} .
$$

The proof of Proposition 1 uses only elementary information-theoretic facts that are established using little more than Jensen's inequality. To get sharper bounds for the case of independent random variables $X_{i}$, in Section 3 we employ a new discrete version of the Fisher information which we call scaled Fisher information, and we prove:

Theorem 1. Poisson Approximation for Independent Variables: If $S_{n}=\sum_{i=1}^{n} X_{i}$ is the sum of $n$ independent binary random variables $X_{1}, X_{2}, \ldots, X_{n}$, with $E\left(S_{n}\right)=\sum_{i=1}^{n} p_{i}=\lambda$,

$$
D\left(P_{S_{n}} \| \operatorname{Po}(\lambda)\right) \leq \frac{1}{\lambda} \sum_{i=1}^{n} \frac{p_{i}^{3}}{1-p_{i}} .
$$

The proof of Theorem 1 combines a natural discrete analog of Stam's subbativity of the Fisher information [35][7], and a recent logarithmic Sobolev inequality of Bobkov and Ledoux [8]. As we discuss extensively in Section 3, Theorem 1 is a significant improvement over Proposition 1, and in certain cases it leads to total variation bounds that are asymptotically optimal up to multiplicative constants in the convergence rate. Moreover, (5) is a nontrivial improvement over existing results, as it gives a bound for the relative entropy and not just the total variation distance.

For an information-theoretic interpretation, consider a triangular array of binary random variables $\left\{\left(X_{1}^{(n)}, X_{2}^{(n)}, \ldots, X_{n}^{(n)}\right), n \geq 1\right\}$, such that the right-hand side of (1) goes to zero as $n \rightarrow \infty$ (as, for example, when the $X_{i}^{(n)}$ are i.i.d. Bernoulli $(\lambda / n)$ ). Then the distribution of $S_{n}$ converges to $\operatorname{Po}(\lambda)$, i.e., $P_{S_{n}}$ comes closer and closer to the "most random" distribution among all those that can be obtained by summing a finite number of Bernoulli random variables: Let $\mathcal{P}(\lambda)$ denote the set of all distributions of sums $S_{n}$ of $n$ independent binary random variables with $E\left(S_{n}\right)=\lambda$, for any finite $n$. Then [19],

$$
H(\operatorname{Po}(\lambda))=\sup \{H(P): P \in \mathcal{P}(\lambda)\} .
$$

So, roughly and somewhat incorrectly speaking, the entropy of $S_{n}$ "increases" to the maximum entropy $H(\operatorname{Po}(\lambda))$ as $n$ grows. This invites a tempting analogy with the second law of thermodynamics, stating that the uncertainty of a physical system increases with time, until the system reaches equilibrium in its maximum entropy state.

Corresponding information-theoretic interpretations and proofs have been given for numerous classical results of proability theory, including the central limit theorem [28][9][4][21], 
the convergence of Markov chains [31][24][6], many large deviations results [12][16][13], the martingale convergence theorem [5][6], and the Hewitt-Savage 0-1 law [29]. See also the powerful comments in [18, pp. 211,215]. Finally, we mention that Johnstone and MacGibbon considered the problem of Poisson convergence from the information theory angle in [22]. Their approach is different from ours, and parallels that in [9][4] for the central limit theorem.

\section{General Bounds in Relative Entropy}

Before giving the proof of Proposition 1 we introduce some some notation and briefly recall two elementary, well-known facts. The first one formalizes the intuitive idea that we cannot do better in a hypothesis test by simply pre-processing the data. Suppose $X$ and $Y$ are random variables with distributions $P$ and $Q$, respectively, let $f$ be an arbitrary function, and write $P^{\prime}, Q^{\prime}$ for the distribution of $f(X)$ and $f(Y)$, respectively. The following "data processing" inequality is an easy consequence of Jensen's inequality [14, Lemma 1.3.11],

$$
D\left(P^{\prime} \| Q^{\prime}\right) \leq D(P \| Q) .
$$

Next, given $X$ and $Y$ with joint distribution $P_{X, Y}$ and marginals $P_{X}$ and $P_{Y}$, let $I(X ; Y)=$ $H(X)-H(X \mid Y)$ denote their mutual information. The "chain rule" is the simple expansion,

$$
D\left(P_{X, Y} \| Q_{X} \times Q_{Y}\right)=D\left(P_{X} \| Q_{X}\right)+D\left(P_{Y} \| Q_{Y}\right)+I(X ; Y),
$$

for any two probability distributions $Q_{X}$ and $Q_{Y}$.

Proof of Proposition 1. If we define $S_{n}^{\prime}=\sum_{i=1}^{n} Z_{i}$, where $Z_{i}$ are independent $\operatorname{Poisson}\left(p_{i}\right)$ random variables, then the distribution $P_{S_{n}^{\prime}}$ of $S_{n}^{\prime}$ is $\operatorname{Po}(\lambda)$ and

$$
\begin{aligned}
D\left(P_{S_{n}} \| \operatorname{Po}(\lambda)\right) & =D\left(P_{S_{n}} \| P_{S_{n}^{\prime}}\right) \\
& \stackrel{(a)}{\leq} D\left(P_{X_{1}, \ldots, X_{n}} \| P_{Z_{1}, \ldots, Z_{n}}\right) \\
& \stackrel{(b)}{=} \sum_{i=1}^{n} D\left(P_{X_{i}} \| \operatorname{Po}\left(p_{i}\right)\right)+\sum_{i=1}^{n-1} I\left(X_{i} ;\left(X_{i+1}, \ldots, X_{n}\right)\right),
\end{aligned}
$$

where $(a)$ follows from the data processing inequality, and $(b)$ follows by applying the chain rule $(n-1)$ times. Using simple calculus we obtain the bound

$$
D(\operatorname{Bern}(p) \| \operatorname{Po}(p))=(1-p) \log \frac{(1-p)}{e^{-p}}+p \log \frac{p}{p e^{-p}} \leq p^{2},
$$

which, applied to each term in the first sum in (6), gives,

$$
\begin{aligned}
D\left(P_{S_{n}} \| \operatorname{Po}(\lambda)\right) & \leq \sum_{i=1}^{n} p_{i}^{2}+\sum_{i=1}^{n-1} I\left(X_{i} ;\left(X_{i+1}, \ldots, X_{n}\right)\right) \\
& =\sum_{i=1}^{n} p_{i}^{2}+\left[\sum_{i=1}^{n} H\left(X_{i}\right)-H\left(X_{1}, X_{2}, \ldots, X_{n}\right)\right],
\end{aligned}
$$

where in the last step we expanded the definition of the mutual informations. 
The first term in the above bound makes precise what we mean by the requirement that "all the $p_{i}$ be small" whereas the second term quantifies their degree of dependence. It is worth noting that this difference between the sum of the entropies of the $X_{i}$ and their joint entropy can also be written as the relative entropy $D\left(P_{X_{1}^{n}} \| P_{X_{1}} \times \cdots \times P_{X_{n}}\right)$ between their joint distribution and the product of their marginals. This expression also admits a natural interpretation as a measure of how far the $X_{i}$ are from being independent.

As indicated in the introduction, although the result of Proposition 1 is generally good enough to prove convergence to the Poisson distribution, for finite $n$ it often gives a suboptimal convergence rate. This is also illustrated in the following two examples.

A Markov Chain. Let $\left\{\left(X_{1}^{(n)}, X_{2}^{(n)}, \ldots, X_{n}^{(n)}\right), n \geq 1\right\}$ be a triangular array of binary random variables such that each row $\left(X_{1}^{(n)}, \ldots, X_{n}^{(n)}\right)$ is a Markov chain with transition matrix

$$
\left(\begin{array}{cc}
\frac{n}{n+1} & \frac{1}{n+1} \\
\frac{n-1}{n+1} & \frac{2}{n+1}
\end{array}\right)
$$

and with each $X_{i}^{(n)}$ having (the stationary) Bernoulli $\left(\frac{1}{n}\right)$ distribution. The convergence of the distribution of $S_{n}=\sum_{i=1}^{n} X_{i}^{(n)}$ to $\mathrm{Po}(1)$ is a well-studied problem; see [10] and the references therein. Applying Proposition 1 (or, equivalently, inequality (7)) in this case translates to

$$
D\left(P_{S_{n}} \| \operatorname{Po}(1)\right) \leq \sum_{i=1}^{n} \frac{1}{n^{2}}+\sum_{i=1}^{n-1} I\left(X_{i}^{(n)} ; X_{i+1}^{(n)}\right)=\frac{1}{n}+(n-1) I\left(X_{1}^{(n)} ; X_{2}^{(n)}\right),
$$

since $I\left(X_{i}^{(n)} ;\left(X_{i+1}^{(n)}, \ldots, X_{n}^{(n)}\right)\right)=I\left(X_{i}^{(n)} ; X_{i+1}^{(n)}\right)$ by the Markov property, and stationarity implies that $I\left(X_{i}^{(n)} ; X_{i+1}^{(n)}\right)=I\left(X_{1}^{(n)} ; X_{2}^{(n)}\right)$. A straightforward calculation yields that

$$
(n-1) I\left(X_{1}^{(n)} ; X_{2}^{(n)}\right)=(n-1)\left[h\left(\frac{1}{n}\right)-h\left(\frac{1}{n+1}\right)\right]+\frac{n-1}{n} h\left(\frac{1}{n+1}\right)-\frac{n-1}{n} h\left(\frac{2}{n+1}\right),
$$

where $h(p)$ denotes the binary entropy function $h(p)=-p \log p-(1-p) \log (1-p)$, and simple calculus shows that all three terms above converge to zero as $n \rightarrow \infty$. In fact, this expression can be bounded above by

$$
h\left(\frac{1}{n+1}\right)+\frac{\log n}{n} \leq 3 \frac{\log n}{n},
$$

where the last inequality holds for all $n \geq 3$, so putting it all together,

$$
D\left(P_{S_{n}} \| \operatorname{Po}(1)\right) \leq 3 \frac{\log n}{n}+\frac{1}{n} .
$$

[A corresponding bound can similarly be derived if instead of stationarity we assume that $X_{1}^{(n)}$ has $p_{1}^{(n)}=E\left(X_{1}^{(n)}\right)<1 / n$.] As mentioned above, although this bound is sufficient to prove that $P_{S_{n}}$ converges to the Poisson distribution, it leads to a convergence rate in total variation of order $\sqrt{(\log n) / n}$, compared to the $O(1 / n)$ bound derived in [3][33][34]. 
A Compound Poisson Approximation Example. Let $X_{1}, \ldots, X_{n}$ be independent Bernoulli random variables with parameters $p_{i}=E\left(X_{i}\right)$, write $\lambda=\sum_{i=1}^{n} p_{i}$, and let $\alpha_{1}, \alpha_{2}, \ldots, \alpha_{n}$ be i.i.d, independent of the $X_{i}$, with distribution

$$
\alpha_{i}=\left\{\begin{array}{cc}
1 & \text { with prob } 1 / 2 \\
2 & \text { with prob } 1 / 2
\end{array}\right.
$$

We will show that the distribution of the sum

$$
S_{n}=\sum_{i=1}^{n} \alpha_{i} X_{i}
$$

is close to the compound Poisson distribution with parameters $(\lambda / 2, \lambda / 2)$, which we denote by $\operatorname{Po}(\lambda / 2, \lambda / 2)$. Recall that if $Z_{1}$ and $Z_{2}$ are i.i.d. Poisson $(\lambda / 2)$ random variables, then $Z=\left(Z_{1}+2 Z_{2}\right)$ has $\operatorname{Po}(\lambda / 2, \lambda / 2)$ distribution. Alternatively, we can write $Z=\sum_{i=1}^{n} Y_{i}$ where the $Y_{i}$ are independent $\operatorname{Po}\left(p_{i} / 2, p_{i} / 2\right)$ random variables. Arguing as before, the data processing inequality and the chain rule imply that

$$
D\left(P_{S_{n}} \| \operatorname{Po}(\lambda / 2, \lambda / 2)\right) \leq D\left(P_{\alpha_{1} X_{1}, \ldots, \alpha_{n} X_{n}} \| P_{Y_{1}, \ldots, Y_{n}}\right)=\sum_{i=1}^{n} D\left(P_{\alpha_{i} X_{i}} \| P_{Y_{i}}\right)
$$

and it is straightforward to calculate

$$
D\left(P_{\alpha_{i} X_{i}} \| P_{Y_{i}}\right) \leq p_{i}^{2}+\left(1-p_{i}\right)\left[p_{i}+\log \left(1-p_{i}\right)\right]-\frac{p_{i}}{2} \log \left(1+p_{i} / 4\right) \leq p_{i}^{2},
$$

so that

$$
D\left(P_{S_{n}} \| \operatorname{Po}(\lambda / 2, \lambda / 2)\right) \leq \sum_{i=1}^{n} p_{i}^{2} .
$$

A general method. Finally, we outline a simple general strategy for approximating the distribution $P_{S_{n}}$ of the sum of $n$ nonnegative-integer-valued random variables $X_{1}, X_{2}, \ldots, X_{n}$ by the distribution of some infinitely divisible discrete random variable $Z$ with $E\left(S_{n}\right)=E(Z)$.

First, use the infinitely divisibility of $P_{Z}$ to represent $Z$ as $Z=\sum_{i=1}^{n} Y_{i}$ where the $Y_{i}$ are independent and have the same distribution as $Z$ but with different parameters. Then apply the data processing inequality and the chain rule as before to obtain

$$
D\left(P_{S_{n}} \| P_{Z}\right) \leq \sum_{i=1}^{n} D\left(P_{X_{i}} \| P_{Y_{i}}\right)+\left[\sum_{i=1}^{n} H\left(X_{i}\right)-H\left(X_{1}, \ldots X_{n}\right)\right],
$$

and finally, estimate the last two terms in above inequality. The first term should be small if the $X_{i}$ are individually small and well-approximated by the corresponding $Y_{i}$, and the second term should be small if the $X_{i}$ are sufficiently weakly dependent. 


\section{Tighter Bounds for Independent Random Variables}

Next we take a different point of view that yields tighter bounds than Proposition 1. Recall that in [22][30][23], the Fisher information of a random variable $X$ with distribution $P$ on $\mathbb{Z}_{+}=\{0,1,2, \ldots\}$, is defined in a way analogous to that for continuous random variables, via

$$
J(X)=E\left[\left(\frac{P(X-1)-P(X)}{P(X)}\right)^{2}\right],
$$

with the convention that $P(-1)=0$. However, as Kagan [23] acknowledges, this definition is really only useful if $X$ is supported on the entire $\mathbb{Z}_{+}$: If $X$ has bounded support then for some $n, P(n)>0$ but $P(n+1)=0$, which implies that $J(X)=\infty$.

Partly in order to avoid this difficulty, we proceed along a different route. Recalling that the Poisson distribution is characterized by the recurrence $\lambda P(x)=(x+1) P(x+1)$ for all $x$, we let the scaled score function of a random variable $X$ with mean $\lambda$ and distribution $P$ on $\mathbb{Z}_{+}$be

$$
\rho_{X}(x)=\frac{(x+1) P(x+1)}{\lambda P(x)}-1, \quad x \in \mathbb{Z}_{+},
$$

and we define the scaled Fisher information of $X$ as

$$
K(X)=\lambda E\left[\rho_{X}(X)^{2}\right] .
$$

From this we easily see that

$$
K(X) \geq 0
$$

with equality iff $\rho_{X}(X)=0$ with probability 1, i.e., iff $X$ is has a $\operatorname{Poisson}(\lambda)$ distribution. Moreover, as we show next, the smaller the value of $K(X)$, the closer $P$ is to the $\operatorname{Poisson}(\lambda)$ distribution. The proof of Proposition 2, given in Section 3.2, is an easy consequence of a recent logarithmic Sobolev inequality of Bobkov and Ledoux [8].

Proposition 2. Relative Entropy and $K(X)$ : If $X$ is a random variable with distribution $P$ on $\mathbb{Z}_{+}$and with $E(X)=\lambda$, then

$$
D(P \| \operatorname{Po}(\lambda)) \leq K(X),
$$

as long as either $P$ has full support (i.e., $P(k)>0$ for all $k$ ), or finite support (i.e., there exists $N \in \mathbb{Z}_{+}$such that $P(k)=0$ for all $\left.k>N\right)$.

Note that from (8) and Pinsker's inequality (2) we have that

$$
\|P-\operatorname{Po}(\lambda)\|_{\mathrm{TV}} \leq \sqrt{2 K(X)} .
$$

We also give a direct proof of (9) in Section 3.2, based on a simple Poincaré inequality for the Poisson measure. 


\subsection{Results}

The main step in the proof of Theorem 1 will be to establish a form of subadditivity for the scaled Fisher information. It is worth noting that in the Gaussian case the Fisher information is also subadditive [35][7], but, in contrast to the present setting, subadditivity alone does not suffice to prove the central limit theorem [4]. Proposition 3 is proved in Section 3.2.

Proposition 3. Subadditivity of Scaled Fisher Information: If $S_{n}=\sum_{i=1}^{n} X_{i}$ is the sum of $n$ independent integer-valued random variables $X_{1}, X_{2}, \ldots, X_{n}$, with means $E\left(X_{i}\right)=p_{i}$ and $E\left(S_{n}\right)=\sum_{i=1}^{n} p_{i}=\lambda$, then

$$
K\left(S_{n}\right) \leq \sum_{i=1}^{n} \frac{p_{i}}{\lambda} K\left(X_{i}\right)
$$

Proof of Theorem 1. If the $X_{i}$ are independent $\operatorname{Bernoulli}\left(p_{i}\right)$ random variables with $\sum_{i=1}^{n} p_{i}=\lambda$, then $K\left(X_{i}\right)=p_{i}^{2} /\left(1-p_{i}\right)$ and Proposition 3 gives

$$
K\left(S_{n}\right) \leq \frac{1}{\lambda} \sum_{i=1}^{n} \frac{p_{i}^{3}}{1-p_{i}}
$$

Combining this with $X=S_{n}$ in Proposition 2 yields inequality (5).

Example 1. If the $X_{i}$ are i.i.d. Bernoulli $(\lambda / n)$ random variables, from Theorem 1 combined with Pinsker's inequality (2) we obtain that for any $\epsilon>0$,

$$
\left\|P_{S_{n}}-\operatorname{Po}(\lambda)\right\|_{\mathrm{TV}} \leq(2+\epsilon) \frac{\lambda}{n}, \quad \text { for } n \geq \lambda / \epsilon .
$$

This is a definite improvement over the earlier $2 \lambda / \sqrt{n}$ bound from (4), and, except for the constant factor, it is asymptotically of the right order; see [3][15] for details.

Example 2. If the $X_{i}$ are i.i.d. Bernoulli $(\mu / \sqrt{n})$ random variables, Theorem 1 together with Pinsker's inequality (2) yield,

$$
\left\|P_{S_{n}}-\operatorname{Po}(\mu \sqrt{n})\right\|_{\mathrm{TV}} \leq \frac{\mu}{\sqrt{n}} \sqrt{\frac{2}{1-\mu / \sqrt{n}}} \approx \frac{\mu}{\sqrt{n}} \sqrt{2},
$$

which is of the same order as the optimal asymptotic rate, as $n \rightarrow \infty$,

$$
\left\|P_{S_{n}}-\operatorname{Po}(\mu \sqrt{n})\right\|_{\mathrm{TV}} \sim \frac{\mu}{\sqrt{n}} \sqrt{1 /(2 \pi e)}
$$

derived in [15].

Example 3. If the $X_{i}$ are Geometric random variables with respective distributions $P_{i}(x)=$ $\left(1-q_{i}\right)^{x} q_{i}, x \geq 0$, then $K\left(X_{i}\right)=\left(1-q_{i}\right)^{2} / q_{i}$. Letting $S_{n}=\sum_{i=1}^{n} X_{i}$ and assuming that $E\left(S_{n}\right)=\sum_{i=1}^{n} \frac{1-q_{i}}{q_{i}}=\lambda$, combining Proposition 3 and the bound (9) yields

$$
\left\|P_{S_{n}}-\operatorname{Po}(\lambda)\right\|_{\mathrm{TV}} \leq \sqrt{\frac{2}{\lambda} \sum_{i=1}^{n} \frac{\left(1-q_{i}\right) 3}{q_{i}^{2}}} .
$$


In particular, taking all the $q_{i}=n /(n+\lambda)$ gives the elegant estimate

$$
\left\|P_{S_{n}}-\operatorname{Po}(\lambda)\right\|_{\mathrm{TV}} \leq \frac{\sqrt{2} \lambda}{\sqrt{n(n+\lambda)}} \leq \sqrt{2} \frac{\lambda}{n} .
$$

To see how tight the result of Proposition 3 is in general, note that the following lower bound of Cramér-Rao type holds: Since for all $a$ and any random variable $S$ with mean $\lambda$ and variance $\sigma^{2}$,

$$
0 \leq \lambda E\left(\rho_{S}(S)-a(S-\lambda)\right)^{2}=K(S)+\lambda\left(a^{2} \sigma^{2}-2 a\left(\frac{\sigma^{2}-\lambda}{\lambda}\right)\right)
$$

choosing $a=\left(\sigma^{2}-\lambda\right) /\left(\sigma^{2} \lambda\right)$, we obtain that

$$
K(S) \geq\left(\sigma^{2}-\lambda\right)^{2} /\left(\sigma^{2} \lambda\right) .
$$

In Example 1 where $S=S_{n}=\sum_{i=1}^{n} X_{i}$ is the sum of $n$ i.i.d. Bernoulli $(\lambda / n)$ random variables, the lower bound (10) coincides with the upper bound given in Proposition 3. Similarly, in Example 3 with all the $q_{i}=n /(n+\lambda)$, the upper bound from Proposition 3 holds with equality. Therefore, any remaining slackness in our bounds comes from either Proposition 2 or Pinsker's inequality.

Finally, in Proposition 4 below we establish a formal connection between relative entropy and the probability distribution $(x+1) P(x+1) / \lambda$ implicitly used in our definition of the scaled Fisher information. It is proved in the next section.

Proposition 4. Let $X$ be an integer-valued random variable with distribution $P$ and mean $\lambda$. If $X$ is the sum of independent Bernoulli random variables, then

$$
D(P \| \operatorname{Po}(\lambda))=\int_{0}^{\infty} D\left(P_{t} \| \widetilde{P}_{t}\right) d t
$$

where $P_{t}(r)=\operatorname{Pr}\left(X_{t}=r\right)$ is the distribution of $X_{t}=X+\operatorname{Po}(t)$ where $\operatorname{Po}(t)$ is an independent Poisson $(t)$ random variable, and $\widetilde{P}_{t}(r)=(r+1) \operatorname{Pr}\left(X_{t}=r+1\right) /(\lambda+t)$. More generally, the same result holds for any random variable $X$ that has $K(X)<\infty$ and satisfies the logarithmic Sobolev inequality of Proposition 2.

This result is reminiscent of the well-known de Bruijn identity, which states that the (differential) relative entropy between a random variable $X$ and a Gaussian with the same variance can be written as a weighted integral of (continuous) Fisher informations of convex combinations of $X$ and an independent $N(0, t)$ random variable; see [11][4]. In a similar vain, if we formally expand the logarithm in the integrand in (11) as a Taylor series, then the first term in the expansion (the quadratic term) turns out to be equal to $K\left(X_{t}\right) / 2(\lambda+t)$. Therefore,

$$
D(P \| \operatorname{Po}(\lambda)) \approx \int_{0}^{\infty} \frac{K(X+\mathrm{Po}(t))}{2(\lambda+t)} d t,
$$

giving an alternative formula to Proposition 2, also relating scaled Fisher information and relative entropy. 


\subsection{Proofs}

Although in several places below we formally divide by a quantity which may be zero, this is taken care of by the usual conventions, $0 \log (0 / a)=0,0 \log (0 / 0)=0$, and $0 \log (a / 0)=\infty$, for any $a>0$.

Proof of Proposition 2. Let $\mathrm{Po}_{\lambda}(k)$ denote the $\operatorname{Po}(\lambda)$ probabilities. In the case when $P$ has full support, the result follows immediately from Corollary 4 of [8], upon considering the function $f(k)=P(k) / \mathrm{Po}_{\lambda}(k), k \geq 0$.

In the case of finite support, for $\epsilon>0$ let $X^{\epsilon}$ have the mixture distribution

$$
P^{\epsilon}=\epsilon \mathrm{Po}_{\lambda}+(1-\epsilon) P .
$$

Then $E\left(X^{\epsilon}\right)=\lambda$ and $P^{\epsilon}$ has full support, so by the previous part,

$$
D\left(P^{\epsilon} \| \operatorname{Po}(\lambda)\right) \leq K\left(X^{\epsilon}\right) .
$$

But since $P(k)=0$ for $k \geq N+1$, then $P^{\epsilon}(k) / \operatorname{Po}(\lambda)(k)=\epsilon$ for those $k$, and letting $\epsilon \downarrow 0$ in the left hand side of (12) we get

$$
D\left(P^{\epsilon} \| \operatorname{Po}(\lambda)\right)=\sum_{k=0}^{N} P^{\epsilon}(k) \log \left[\frac{P^{\epsilon}(k)}{\operatorname{Po}_{\lambda}(k)}\right]+\operatorname{Pr}\{\operatorname{Po}(\lambda)>N\} \epsilon \log \epsilon \rightarrow D(P \| \operatorname{Po}(\lambda)) .
$$

Moreover,

$$
\frac{(k+1) P^{\epsilon}(k+1)}{\lambda P^{\epsilon}(k)}=1, \quad k \geq N+1
$$

so

$$
K\left(X^{\epsilon}\right)=\sum_{k=0}^{N} P^{\epsilon}(k)\left[\frac{(k+1) P^{\epsilon}(k+1)}{\lambda P^{\epsilon}(k)}-1\right]^{2} \rightarrow K(X),
$$

as $\epsilon \downarrow 0$, and this completes the proof.

Next we prove the bound in (9) using a classical Poincaré inequality for the Poisson distribution. We actually establish the following (apparently stronger) bound for the Hellinger distance $\|P-\operatorname{Po}(\lambda)\|_{H}$ between $P$ and $\operatorname{Po}(\lambda)$ :

$$
\|P-\operatorname{Po}(\lambda)\|_{\mathrm{TV}}^{2} \leq\|P-\operatorname{Po}(\lambda)\|_{H}^{2} \leq 2 K(X) .
$$

Proof of (9). For any function $f: \mathbb{Z}_{+} \rightarrow \mathbb{R}$, define $\Delta f(x)=f(x+1)-f(x)$. It is well-known that, writing $\operatorname{Po}_{\lambda}(x)$ for the Poisson $(\lambda)$ probabilities, then for all functions $g$ in $L^{2}(q)$,

$$
\sum_{x} \operatorname{Po}_{\lambda}(x)(g(x)-\mu)^{2} \leq \lambda \sum_{x} \operatorname{Po}_{\lambda}(x)(\Delta g(x))^{2},
$$

where $\mu=\sum_{x} g(x) \operatorname{Po}_{\lambda}(x)$ is the mean of $g$ under $\operatorname{Po}(\lambda)$; see for example Klaassen [25].

Using the simple fact that

$$
(\sqrt{u}-1)^{2} \leq(\sqrt{u}-1)^{2}(\sqrt{u}+1)^{2}=(u-1)^{2}, \quad \text { for all } u \geq 0,
$$


we get that

$$
K(X)=\lambda \sum_{x} P(x)\left(\frac{P(x+1) \mathrm{Po}_{\lambda}(x)}{\mathrm{Po}_{\lambda}(x+1) P(x)}-1\right)^{2} \geq \lambda \sum_{x} P(x)\left(\sqrt{\frac{P(x+1) \mathrm{Po}_{\lambda}(x)}{\mathrm{Po}_{\lambda}(x+1) P(x)}}-1\right)^{2},
$$

and applying (13) to the function $g(x)=\sqrt{P(x) / \mathrm{Po}_{\lambda}(x)}$ we obtain,

$$
\begin{aligned}
K(X) & \geq \lambda \sum_{x} \operatorname{Po}_{\lambda}(x)\left(\sqrt{\frac{P(x+1)}{\operatorname{Po}_{\lambda}(x+1)}}-\sqrt{\frac{P(x)}{\operatorname{Po}_{\lambda}(x)}}\right)^{2} \\
& \geq \sum_{x} \operatorname{Po}_{\lambda}(x)\left(\sqrt{\frac{P(x)}{\operatorname{Po}_{\lambda}(x)}}-\mu\right)^{2}=1-\mu^{2},
\end{aligned}
$$

where $\mu=\sum_{x} \sqrt{P(x) \operatorname{Po}_{\lambda}(x)}$. Therefore, the Hellinger distance $\|P-\operatorname{Po}(\lambda)\|_{H}$ satisfies

$$
\|P-\operatorname{Po}(\lambda)\|_{H}^{2}=(2-2 \mu) \leq 2\left(1-\mu^{2}\right) \leq 2 K(X),
$$

and since $\|P-\operatorname{Po}(\lambda)\|_{\mathrm{TV}} \leq \sqrt{\|P-\operatorname{Po}(\lambda)\|_{H}}$ (see, e.g., [32, p. 360]) the result follows.

For the proof of Proposition 3, as in the case of normal convergence in Fisher information, we exploit the theory of $L^{2}$ spaces and the fact that scaled score functions of sums are conditional expectations (projections) of the original scaled score functions.

Lemma. Convolution: If $X$ and $Y$ are nonnegative integer-valued random variables with probability distributions $P$ and $Q$ and means $p$ and $q$, respectively, then,

$$
\rho_{X+Y}(z)=E\left[\alpha_{X} \rho_{X}(X)+\alpha_{Y} \rho_{Y}(Y) \mid X+Y=z\right],
$$

where $\alpha_{X}=p /(p+q), \alpha_{Y}=q /(p+q)$.

Proof. Writing $F(z+1)=\sum_{x} P(x) Q(z-x+1)$ for the distribution of $X+Y$, we have,

$$
\begin{aligned}
\rho_{X+Y}(z)= & \sum_{x} \frac{(z+1) P(x) Q(z-x+1)}{(p+q) F(z)}-1 \\
= & \sum_{x}\left[\frac{x P(x) Q(z-x+1)}{(p+q) F(z)}+\frac{(z-x+1) P(x) Q(z-x+1)}{(p+q) F(z)}\right]-1 \\
= & \alpha_{X}\left[\sum_{x} \frac{x P(x)}{p P(x-1)} \frac{P(x-1) Q(z-x+1)}{F(z)}-1\right] \\
& +\alpha_{Y}\left[\sum_{x} \frac{(z-x+1) Q(z-x+1)}{q Q(z-x)} \frac{P(z)-x)}{F(z)}-1\right] \\
\stackrel{(a)}{=} & \sum_{x} \frac{P(x) Q(z-x)}{F(z)}\left[\alpha_{X} \rho_{X}(x)+\alpha_{Y} \rho_{Y}(z-x)\right]
\end{aligned}
$$

as required, where $(a)$ follows by moving $x$ to $(x+1)$ in the first sum. 
Proof of Proposition 3. It suffices to prove the case $n=2$. By the Lemma,

$$
\begin{aligned}
0 & \leq E\left[\frac{p_{1}}{\lambda} \rho_{X_{1}}\left(X_{1}\right)+\frac{p_{2}}{\lambda} \rho_{X_{2}}\left(X_{2}\right)-\rho_{X_{1}+X_{2}}\left(X_{1}+X_{2}\right)\right]^{2} \\
& =E\left[\frac{p_{1}}{\lambda} \rho_{X_{1}}\left(X_{1}\right)+\frac{p_{2}}{\lambda} \rho_{X_{2}}\left(X_{2}\right)\right]^{2}-E\left[\rho_{X_{1}+X_{2}}\left(X_{1}+X_{2}\right)\right]^{2}
\end{aligned}
$$

therefore, noting that $E\left[\rho_{X}(X)\right]=0$ for any random variable $X$,

$$
\begin{aligned}
K\left(X_{1}+X_{2}\right) & =\left(p_{1}+p_{2}\right) E\left[\rho_{X_{1}+X_{2}}\left(X_{1}+X_{2}\right)\right]^{2} \\
& \leq \lambda E\left[\frac{p_{1}}{\lambda} \rho_{X_{1}}\left(X_{1}\right)+\frac{p_{2}}{\lambda} \rho_{X_{2}}\left(X_{2}\right)\right]^{2} \\
& =\frac{p_{1}}{\lambda}\left(p_{1} E\left[\rho_{X_{1}}\left(X_{1}\right)\right]^{2}\right)+\frac{p_{2}}{\lambda}\left(p_{2} E\left[\rho_{X_{2}}\left(X_{2}\right)\right]^{2}\right) \\
& =\frac{p_{1}}{\lambda} K\left(X_{1}\right)+\frac{p_{2}}{\lambda} K\left(X_{2}\right),
\end{aligned}
$$

as claimed.

Proof of Proposition 4. Assume for the moment that the relative entropy between $P_{t}$ and $\operatorname{Po}(\lambda+t)$ tends to zero as $t \rightarrow \infty$ (this will be established below). Then we can write $D(P \| \operatorname{Po}(\lambda))$ as the integral

$$
\begin{aligned}
D(P \| \operatorname{Po}(\lambda)) & =-\int_{0}^{\infty} \frac{\partial}{\partial t} D\left(P_{t} \| \operatorname{Po}(\lambda+t)\right) d t \\
& =-\int_{0}^{\infty} \frac{\partial}{\partial t}\left((\lambda+t)-E\left[X_{t} \log (\lambda+t)\right]+E\left[\log \left(X_{t} !\right)\right]-H\left(X_{t}\right)\right) d t \\
& =\int_{0}^{\infty}\left(\log (\lambda+t)-\frac{\partial}{\partial t} E\left[\log \left(X_{t} !\right)\right]+\frac{\partial}{\partial t} H\left(X_{t}\right)\right) d t .
\end{aligned}
$$

Since the probabilities $P_{t}$ satisfy a differential-difference equation, $\frac{\partial P_{t}}{\partial t}(x)=P_{t}(x-1)-P_{t}(x)$, we have,

$$
\frac{\partial}{\partial t} E\left[\log \left(X_{t}\right) !\right]=\sum_{r} \frac{\partial P_{t}}{\partial t}(r) \log r !=\sum_{r}\left(P_{t}(r-1)-P_{t}(r)\right) \log r !=E \log \left(X_{t}+1\right),
$$

and similarly,

$$
\frac{\partial}{\partial t} H\left(X_{t}\right)=-\sum_{r} \frac{\partial P_{t}}{\partial t}(r) \log P_{t}(r)=\sum_{r} P_{t}(r) \log \left(\frac{P_{t}(r)}{P_{t}(r+1)}\right) .
$$

Substituting these two expressions in the expansion of $D(P \| \operatorname{Po}(\lambda))$ the result follows.

Finally it remains to establish our initial assumption. If $X$ is the sum of independent Bernoulli random variables then it has finite support and Proposition 2 holds; moreover, $K(X)$ is easily seen to be finite by Proposition 3. More generally, using Propositions 2 and 3 we have

$$
D\left(P_{t} \| \operatorname{Po}(\lambda+t)\right) \leq K(X+\operatorname{Po}(t)) \leq \frac{\lambda}{\lambda+t} K(X) \rightarrow 0,
$$

as $t \rightarrow \infty$, as required. 


\section{Acknowledgments}

Interesting conversations with Persi Diaconis, Stu Geman and Matt Harrison are greatfully acknowledged. We also thank the Associate Editor and the two referees for their useful comments on an earlier version of the manuscript.

\section{References}

[1] R. Arratia, L. Goldstein, and L. Gordon. Two moments suffice for Poisson approximations: the Chen-Stein method. Ann. Probab., 17:9-25, 1989.

[2] R.R. Bahadur. Some Limit Theorems in Statistics. SIAM, Philadelphia, PA, 1971.

[3] A.D. Barbour, L. Holst, and S. Janson. Poisson Approximation. The Clarendon Press Oxford University Press, New York, 1992.

[4] A.R. Barron. Entropy and the central limit theorem. Ann. Probab., 14:336-342, 1986.

[5] A.R. Barron. Information theory and martingales. In Int. Symp. Inform. Theory, Budapest, Hungary, 1991.

[6] A.R. Barron. Limits of information, Markov chains, and projection. In Int. Symp. Inform. Theory, Sorrento, Italy, 2000.

[7] N.M. Blachman. The convolution inequality for entropy powers. IEEE Trans. Information Theory, 11:267-271, 1965.

[8] S.G. Bobkov, and M. Ledoux. On modified logarithmic Sobolev inequalities for Bernoulli and Poisson measures. J. Funct. Anal., 156:347-365, 1998.

[9] L.D. Brown. A proof of the central limit theorem motivated by the Cramér-Rao inequality. In Statistics and probability: essays in honor of C. R. Rao, pages 141-148. North-Holland, Amsterdam, 1982.

[10] V. Cekanavičius. On the convergence of Markov binomial to Poisson distribution. Statist. Probab. Lett., 58(1):83-91, 2002.

[11] T.M. Cover and J.A. Thomas. Elements of Information Theory. J. Wiley, New York, 1991.

[12] I. Csiszár. Sanov property, generalized $I$-projection and a conditional limit theorem. Ann. Probab., 12(3):768-793, 1984.

[13] I. Csiszár, T.M. Cover, and B.S. Choi. Conditional limit theorems under Markov conditioning. IEEE Trans. Inform. Theory, 33(6):788-801, 1987.

[14] I. Csiszár and J. Körner. Information Theory: Coding Theorems for Discrete Memoryless Systems. Academic Press, New York, 1981. 
[15] P. Deheuvels and D. Pfeifer. A semigroup approach to Poisson approximation. Ann. Probab., 14(2):663-676, 1986.

[16] A. Dembo and O. Zeitouni. Large Deviations Techniques And Applications. SpringerVerlag, New York, second edition, 1998.

[17] R. Durrett. Probability: Theory and Examples. Duxbury Press, Belmont, CA, second edition, 1996.

[18] B.V. Gnedenko and V.Yu. Korolev. Random Summation: Limit Theorems and Applications. CRC Press, Boca Raton, FL, 1996.

[19] P. Harremoës. Binomial and Poisson distributions as maximum entropy distributions. IEEE Trans. Inform. Theory, 47(5):2039-2041, 2001.

[20] P. Harremoës. The information topology. In Proc. of the IEEE International Symposium on Inform. Theory, page 431, Lausanne, Switzerland, 2002.

[21] O. Johnson. Entropy inequalities and the central limit theorem. Stochastic Process. Appl., 88:291-304, 2000.

[22] I.M. Johnstone and B. MacGibbon. Une mesure d'information caractérisant la loi de Poisson. In Séminaire de Probabilités, XXI, pages 563-573. Springer, Berlin, 1987.

[23] A. Kagan. Letter to the editor: "A discrete version of the Stam inequality and a characterization of the Poisson distribution" [J. Statist. Plann. Inference 92 (2001), no. 1-2, 7-12. J. Statist. Plann. Inference, 99(1):1, 2001.

[24] D.G. Kendall. Information theory and the limit-theorem for Markov chains and processes with a countable infinity of states. Ann. Inst. Statist. Math., 15:137-143, 1963.

[25] C.A.J. Klaassen. On an inequality of Chernoff. Ann. Probab., 13(3):966-974, 1985.

[26] S. Kullback. Information Theory and Statistics. Dover Publications Inc., Mineola, NY, 1997. Reprint of the second (1968) edition.

[27] L. Le Cam. An approximation theorem for the Poisson binomial distribution. Pacific J. Math., 10:1181-1197, 1960.

[28] Ju.V. Linnik. An information-theoretic proof of the central limit theorem with Lindeberg conditions. Theor. Probability Appl., 4:288-299, 1959.

[29] N. O'Connell. Information-theoretic proof of the Hewitt-Savage zero-one law. Technical report, Hewlett-Packard Laboratories, Bristol, UK, June 2000. Available at http://www.hpl.hp.com/techreports/2000/HPL-BRIMS-2000-18.html

[30] V. Papathanasiou. Some characteristic properties of the Fisher information matrix via Cacoullos-type inequalities. J. Multivariate Anal., 44(2):256-265, 1993. 
[31] A. Rényi. On measures of entropy and information. In Proc. 4th Berkeley Sympos. Math. Statist. and Prob., Vol. I, pages 547-561. Univ. California Press, Berkeley, Calif., 1961.

[32] L. Saloff-Coste. Lectures on finite Markov chains. In Lectures on probability theory and statistics (Saint-Flour, 1996), volume 1665 of Lecture Notes in Math., pages 301-413. Springer, Berlin, 1997.

[33] R.F. Serfozo. Compound Poisson approximations for sums of random variables. Ann. Probab., 14:1391-1398, 1986.

[34] R.F. Serfozo. Correction to: "Compound Poisson approximations for sums of random variables". Ann. Probab., 16:429-439, 1988.

[35] A.J. Stam. Some inequalities satisfied by the quantities of information of Fisher and Shannon. Information and Control, 2:101-112, 1959. 
Ioannis Kontoyiannis was born in Athens, Greece, in 1972. He received the B.Sc. degree in mathematics in 1992 from Imperial College (University of London), and in 1993 he obtained a distinction in Part III of the Cambridge University Pure Mathematics Tripos. In 1997 he received the M.S. degree in statistics, and in 1998 the Ph.D. degree in electrical engineering, both from Stanford University. Between June and December 1995 he worked at IBM Research, on a NASA-IBM satellite image processing and compression project. From June 1998 to August 2001 we was an Assistant Professor with the Department of Statistics at Purdue University (and also, by courtesy, with the Department of Mathematics, and the School of Electrical and Computer Engineering). Since August 2000 he has been an Assistant, then Associate Professor, with the Division of Applied Mathematics and with the Department of Computer Science at Brown University. In 2002 he was awarder the Manning endowed assistant professorship and in 2004 he was awarded a Sload Foundation Research Fellowship. His research interests include data compression, applied probability, information theory, statistics, and mathematical biology.

Peter Harremoës (M'00) was born in 1964. He recieved the M.Sc. degree in mathematics as major and archaeology as minor from University of Copenhagen, Copenhagen, Denmark, in 1989 and the Ph.D. degree from Roskilde University, Roskilde, Denmark, in 1993. Since 2001, he had a Postdoctorial position in the Mathematical Department, University of Copenhagen. During 2002-2003, he was Research Fellow at Zentrum fr Interdisziplinre Forshung, University of Bielefeld, Bielefeld, Germany.

Oliver Johnson received MA and PhD degrees from Queens' College, Cambridge University, UK, in 1999 and 2000 respectively. He is now a Clayton Fellow of Christ's College, Cambridge University, and Max Newman Research Fellow of the Statslab, DPMMS, Cambridge University. He works on problems in probability theory, including limit theorems, quantum information and statistical physics. 